\title{
Vibrational Impact of an Elastic Link
}

\author{
Dan B. Marghitu and Malcolm J. Crocker \\ Department of Mechanical Engineering, Auburn University, AL 36849, U.S.A.
}

\begin{abstract}
An experimental analysis was conducted to study the rebound velocities of a freely dropped bar on a large external surface. The low velocity collision occured in the presence of a frictional impact. A 3D motion analysis system was used to capture the kinematic data. Tests were performed for central and oblique collisions. A spatial theoretical model is presented for the impact with friction of a flexible body. This model consists of a system of nonlinear differential equations which considers the multiple collisions as well as frictional effects at the contacting end, and allows one to predict the rigid and elastic body motion after the impact. The model uses a dry coefficient of friction, a nonlinear contact force, and the sound radiation from the impact. Analytical and experimental results were compared to establish the accuracy of the model.
\end{abstract}

\section{INTRODUCTION}

The propagation of impact-induced elastic waves has been the subject of many investigations. The latest development has been in the area of the effect of elastic vibrations on the post-impact velocities of slender bars in motion. Shabana and $\mathrm{Gau}^{1-3}$ have examined the effect of the topological change in the propagation of longitudinal elastic waves in mechanical systems. The application of the analysis presented is demonstrated using a rotating rod that is subject to an axial impact by a rigid mass moving with a constant velocity. On the experimental front, Yigit et al. ${ }^{4}$ verified the validity of using the algebraic generalized impulse momentum equations of a radially rotating beam, transversally impacting an external surface. Their conclusion was that the momentum balance method and an empirical coefficient of restitution can be used with confidence in the impact of a radially rotating beam. A spring-dashpot model for the dynamics of a radially rotating beam with impact was studied by the same authors ${ }^{5}$. A study focusing on the vibrations, multiple collisions, and slip reversal at the contacting point has also been conducted ${ }^{6}$. Impacts that appear to be single to the naked eye can consist in reality of several collisions in rapid sequence. The existence of multiple collisions has been recognized by $\mathrm{Mason}^{7}$, Goldsmith $^{8}$, and observed experimentally by Yigit et al., ${ }^{4,5}$

The objective of the present article is to develop a procedure that can be followed to solve the frictional threedimensional collision of unconstrained slender bars with a massive external surface. An analytical continuous model has been developed and validated by comparing theoretical predictions with experimental results.

\section{EXPERIMENTAL DESIGN}

The main goal of the present investigation was to study the spatial collisions of bars with massive external surfaces. For this purpose an experimental set-up was developed.

The impact was achieved by dropping a bar from a vertical height $h$ on a massive block (Fig. 1). A simple electromechanical device was used to release the bar above the impact surface. The initial orientations of the bar could be set mechanically on the device. The kinematic data were acquired by means of a motion analysis system from which the pre-collision and post-collision velocities were determined. The experimentally obtained kinematic data were limited to the general rigid body motion of the bar. The presence of multiple collisions was established by means of an oscilloscope and a finite number of distinct impacts were detected visibly. The sound pressure was measured with a condenser microphone and a preamplifier. The output of the preamplifier was analyzed with a fast-Fourier-transform analyzer. The analyzer recorded the sound pressure as a time record. Finally, these data were saved on a personal computer. The individual components in the setup are described below.

\subsection{Motion Analysis System}

The principal component in the setup is a WATSMART, Northern Digital three-dimensional digitizing and motion analysis system. This system is comprised of a pair of infrared cameras linked to a data acquisition and digitizing unit, and is controlled through a computer. Active infrared emitting diodes attached to the target object (flexible link) are tracked by the cameras through a pre-calibrated space. This system has a sampling rate of $4200 \mathrm{~Hz}$.

\subsection{Signal Analyzer}

In order to detect the multiple collisions during impact a signal analyzer was used. The particular unit was a $H E W L$ ETT-PACKARD $35665 A$ dynamic signal analyzer with a frequency range that extends to $100 \mathrm{kHz}$. This analyzer was connected to both the slender bar and the impact surface and the number of contact events and the their duration was recorded. The contact between the bar and the surface was used as a switch in an electrical circuit in order to sense the duration of collision. The contact events during the collision were detected by capturing the voltage generated in the circuit with a triggering oscilloscope.

\subsection{Sound Analyzer}

As the bar hits the heavy collision surface an impact sound is generated. To measure and examine the sound a BRUEL\&KJAER $4135+2633$ preamplifier and a BRUEL\& KJAER 2032 fast-Fourier-transform analyzer were used. To capture the impact sound in a short period, it was necessary 